La revue La revue pour l'histoire du CNRS

POUR L'HISTOIRE DU CNRS $\quad 21 \mid 2008$

Entre mémoire et oubli

\title{
La mémoire en héritage
}

Réflexions sur l'histoire des traces mnésiques

Jean-Claude Dupont

\section{(2) OpenEdition}

Journals

Édition électronique

URL : https://journals.openedition.org/histoire-cnrs/7592

DOI : $10.4000 /$ histoire-cnrs.7592

ISSN : 1955-2408

Éditeur

CNRS Éditions

Édition imprimée

Date de publication : 3 juillet 2008

ISBN : 978-2-271-06675-6

ISSN : $1298-9800$

Référence électronique

Jean-Claude Dupont, "La mémoire en héritage », La revue pour I'histoire du CNRS [En ligne], 21 | 2008, mis en ligne le 03 juillet 2010, consulté le 20 mai 2021. URL : http://journals.openedition.org/histoirecnrs/7592 ; DOl : https://doi.org/10.4000/histoire-cnrs.7592

Ce document a été généré automatiquement le 20 mai 2021

Comité pour l'histoire du CNRS 


\title{
La mémoire en héritage
}

\author{
Réflexions sur l'histoire des traces mnésiques
}

Jean-Claude Dupont

1 La matérialisation des processus mnésiques, l'identification, et la localisation de ces traces mémorielles constituent une part importante du programme de recherche des neurosciences contemporaines ${ }^{1}$. Leur signification est âprement discutée ${ }^{2}$. Plus que ces développements, ce qui nous retiendra ici est que, de leur côté, les historiens se sont consacrés à démontrer l'extrême ancienneté de la notion de trace. La description du fonctionnement de la mémoire, qui se prétend aujourd'hui débarrassée de toute métaphore ou de toute analogie, unique résultat d'une reconstruction des différentes fonctions mnésiques et de la recherche patiente de «corrélats » organiques par les moyens les plus sophistiqués, fait suite à la permanence de métaphores dont la réalité, et souvent la valeur heuristique, sont désormais en effet historiquement bien attestées ${ }^{3}$.

De la métaphore à la trace mnésique

2 Sur la base de l'idée grecque du tupos, la culture occidentale va ainsi utiliser d'emblée deux images complémentaires pour exprimer l'inscription corporelle des souvenirs : la mémoire comme jeu de tablettes de cires où sont inscrites des informations, et la mémoire comme magasin ou inventaire. Sur ces modèles, les discours sur la mémoire s'engagent dans la voie d'une incarnation toujours plus grande, voire d'une cérébralisation de la mémoire, en intention du moins, compte tenu de l'insuffisance de l'instrumentation et des modèles théoriques. Depuis les métaphores grecques, l'idée que la mémoire doit être associée à une modification physique et corporelle parcourt en effet toute l'histoire de la pensée occidentale, des observations cliniques des médecins renaissants jusqu'aux physiologies des Lumières. Le médecin David Hartley va par exemple combiner la théorie vibratoire de Newton avec l'associationniste de Locke pour formuler une théorie organique de la mémoire (1749).

L'apport de la physique et la chimie

3 L'idée y est conçue comme une modification de l'état vibratoire d'une portion du système nerveux, la « composition » des ondes est le corrélat physique de l'association, 
et l'impression des ondes, qui suppose que la matière nerveuse possède des propriétés plastiques, est celui de la mémoire.

4 À partir du XVIII siècle se multiplient de telles tentatives de compréhension des traces mémorielles par des analogies avec des phénomènes chimiques ou physiques tels qu'ils sont alors inventés: les phénomènes vibratoires et de résonance, mais aussi les mouvements ou rétentions du fluide (nerveux), le stockage de l'énergie électrique, le stockage magnétique, l'hystérésis, l'élasticité, le principe d'inertie, la cristallisation, la phosphorescence, et bien plus tard les processus auto-catalytiques et de réarrangement moléculaire.

5 La valeur de cette tradition de compréhension variée de la mémoire psychique comme phénomène physiologique, de théories organiques de la mémoire, est diversement appréciée. David Krell analyse les formes successivement prises par les discours physiques de la mémoire chez Platon, Aristote, Hobbes, Locke, Descartes, Hartley, Freud... en nous expliquant qu'elles échouent à la véritable compréhension du phénomène $e^{4}$. L'appréciation de John Sutton est tout autre, qui croit déceler une continuité de la notion de traces mémorielles depuis Descartes vers le connexionnisme d'aujourd'hui, en une sorte de marche triomphale ${ }^{5}$.

6 Quoi qu'il en soit, même s'il faut considérer la permanence de la notion de trace, ces appréciations négatives ou louangeuses ne sont pas d'un grand secours à l'historien lorsqu'il lui faut plutôt aborder la question si cruciale des commencements, de cette rupture ou de ce point d'inflexion qui va faire de la notion de trace un concept opératoire. Il lui faut identifier ces conditions intellectuelles et matérielles qui permettent la transition d'une science désirée à une science effective.

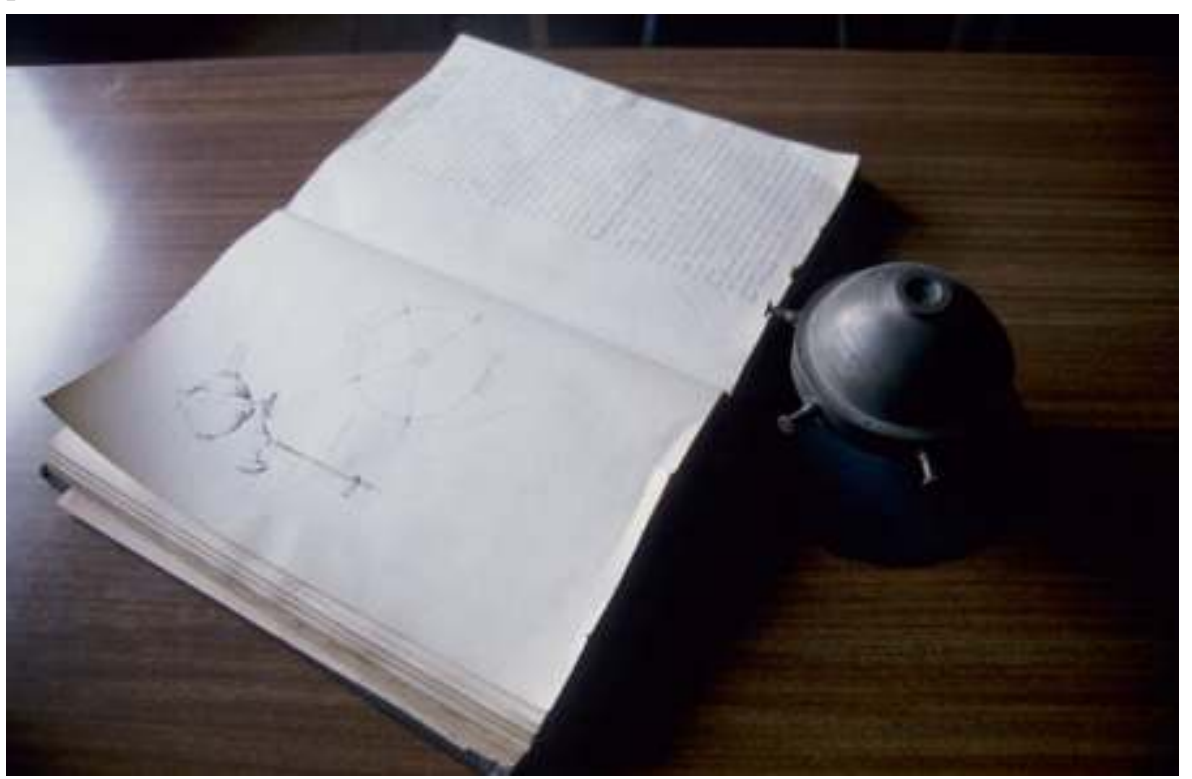

Cahier de travaux de pratiques de Paul Langevin et actinomètre de Féry. (c) CNRS Photothèque/ Édouard Golbin

La mémoire organique

7 Ces conditions ici sont nombreuses et restent encore à reconstituer. Elles semblent concerner avant tout les sciences du cerveau: la théorie du neurone, la neurophysiologie de l'apprentissage, la reconnaissance de la plasticité cérébrale sont autant d'étapes qui vont donner une consistance matérielle ou une réalité cérébrale à la notion de trace. Mais ce qui importe aussi est le concept de rupture : pour que s'impose 
l'idée de trace mémorielle au sens actuel, il a fallu parallèlement rejeter l'idée d'une mémoire organique générale. Le dernier quart du XIX siècle voit naître ce concept de mémoire organique, à la jonction de la biologie et de la psychologie, identifiant, au-delà d'un simple rapprochement, mémoire héréditaire et mémoire mentale.

8 Il faut rappeler l'extraordinaire extension de la mémoire organique dans les milieux scientifiques, médicaux et littéraires de l'époque. En dehors de Ribot, c'est le physiologiste Viennois Ewald Hering qui l'exprime le mieux dans sa fameuse dissertation sur "La mémoire comme fonction universelle de la matière organisée " (1870). La mémoire doit être considérée comme une capacité inhérente à notre substance cérébrale et obéir à des lois physiques. Elle réalise une unification du présent d'un organisme avec son passé non seulement durant la durée de sa vie mais aussi à travers les générations. Elle s'étend à toutes les cellules corporelles, incluant les cellules germinales, et devient ainsi le support organique de l'hérédité des caractères acquis. Alors que Hering limitait la mémoire organique aux produits cellulaires, Ernst Haeckel l'étend à toutes les molécules complexes, même inorganiques.

La thèse de l'hérédité

9 La mémoire organique explique donc non seulement l'hérédité, mais aussi, par exemple, la cristallisation. En vertu de la loi de la récapitulation, c'est d'ailleurs l'ensemble de la phylogénie et de l'ontogénie qui est susceptible de relever de la mémoire organique, qui devient ainsi principe naturel unifiant une théorie de l'hérédité, une théorie de l'évolution, et une théorie psychologique. En pathologie, le concept de mémoire organique transparaît dans la théorie de la dégénérescence de Benedict Morel et Valentin Magnan. Les troubles des arriérés sont à rechercher dans l'hérédité, ou dans les affections acquises du jeune âge (alcoolisme, misère, etc.). Pour que la dégénérescence soit progressive, chaque individu ajoute à son atavisme ses propres expériences. L'influence de l'hérédité pathologique se retrouve dans la thèse du criminel de Lombroso, et a été popularisée par le naturalisme en tant qu'école littéraire (Zola).

10 Hering n'est d'ailleurs le représentant que de la première génération des théoriciens de la mémoire organique: une seconde génération (Francis Darwin, Eugenio Rignano, Richard Semon) va prétendre donner au concept de mémoire organique une base scientifique plus sûre que celle que lui avaient donnée Hering ou Ribot. Le procès de l'hérédité de l'acquis et les progrès de la génétique morgano-mendélienne vont signer le déclin progressif de l'hérédité mnémonique. Impasse heureuse puisqu'elle a permis de libérer la mémoire humaine pour un espace qui restait à construire.

11 Mais cette dynamique n'est pas suffisante pour comprendre l'émergence scientifique de l'objet mémoire. La fin du XIX ${ }^{\mathrm{e}}$ siècle représente ce vrai commencement des sciences de la mémoire de l'homme individuel d'abord parce que, dans le champ du savoir, la situation de la mémoire change alors, tant au niveau des écrits que des pratiques. La mémoire humaine ne pouvait plus se contenter depuis longtemps d'être l'élément, plus ou moins noble, d'une philosophie générale de l'esprit ou de la connaissance, ou au contraire relever d'une simple technique de manipulation mnémonique destinée à l'améliorer.

12 Nous avons vu dans quelles conditions la mémoire ne pouvait pas relever non plus d'une biologie dont elle constituerait le fondement, en une fantasmatique mémoire organique. Elle ne pouvait plus même être inclue, plus ou moins harmonieusement, dans une élégante et spéculative psychologie générale des facultés. 
L'objet mémoire

La mémoire doit désormais se constituer comme un objet dont l'étude devient en ellemême une fin suffisante, c'est-à-dire comme un véritable objet de science, objet travaillé par différentes disciplines en construction: psychologie, psychiatrie, neurologie. Bien que les écrits, même spécialisés, sur la mémoire aient été antérieurement fort nombreux, deviennent en effet seulement au cours du XIX ${ }^{\mathrm{e}}$ siècle possibles et clairement repérables des stratégies spécifiques visant à élucider des fonctionnements mnésiques. C'est ainsi qu'initialement surtout concentrée sur les questions perceptives, la psychologie expérimentale naissante s'organise autour d'elle, tandis qu'en psychiatrie se développe une taxinomie clinique des amnésies, et que les biologistes élaborent de nouvelles hypothèses psychophysiologiques. La mémoire, frénétiquement observée, expérimentée, disséquée, est en mesure d'être naturalisée dirait-on aujourd'hui. Quelle que soit l'ancienneté des théories physiologiques de la mémoire et des métaphores évoquées plus haut, l'histoire des sciences de la mémoire, entendues de la mémoire comme objet travaillé par différentes communautés en compétition, ne couvre guère bien qu'un siècle ou deux ${ }^{6}$.

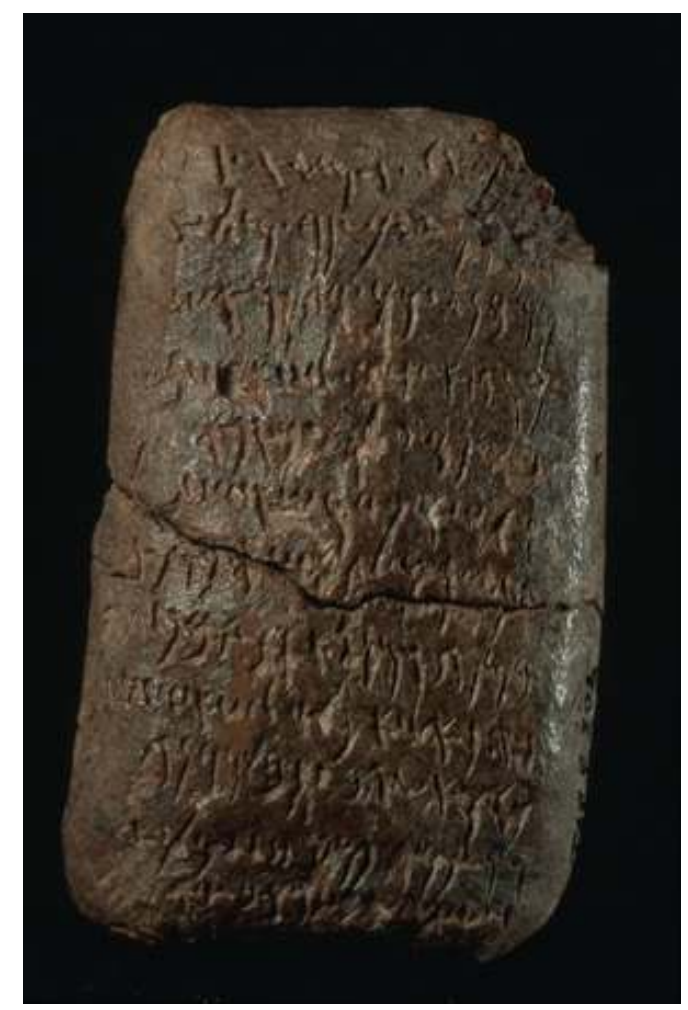

Le plus long document araméen sur tablette d'argile jamais trouvé (VIle siècle av. J.-C.). Tell Shioukh Faouqâni (Syrie, vallée de l'Euphrate). ( C CNRS Photothèque/Luc Bachelot

Les supports biologiques de la mémoire

Plus qu'une rupture, la notion de trace ne devient donc opératoire, ou ne prend corps, qu'à l'issue d'une convergence qui est loin de concerner les seules sciences du cerveau. Les difficultés sont redoutables: la psychologie est parcourue tout au long de son histoire par de multiples et récurrentes dichotomies (un/multiple, normal/ pathologique, fonction/structure, inconscient/conscient) révélant la complexité de la problématique des fonctions psychiques et de la mémoire en particulier. La notion biologique de trace va devoir intégrer ces difficultés, se confronter à cette complexité révélée par les psychologues et les psychiatres. À partir de ces commencements, il faut 
comprendre le développement de théories de la mémoire, et en montrer à chaque fois l'articulation dans le champ biologique: comment ces théories abandonnent progressivement les analogies, fussent-elles biologiques (avec les phénomènes sensoriels, réflexes, hormonaux, immunologiques), puis abandonnent l'espoir illusoire de l'identification de molécules de mémoire, pour inventer des modèles spécifiques, c'est-à-dire devenir de véritables théories organiques de la mémoire. Ce développement n'est effectif que dans la deuxième moitié $\mathrm{du} \mathrm{XX}^{\mathrm{e}}$ siècle : le plus connu du grand public est représenté par les recherches d'Eric Kandel concernant les supports neurobiologiques de la mémoire, fondées sur le concept ancien de réseau neuronal plastique et mettant en jeu les mécanismes moléculaires de la neurotransmission. Georges Chapouthier a bien monté le caractère interdisciplinaire et l'importante contribution française en ce domaine?

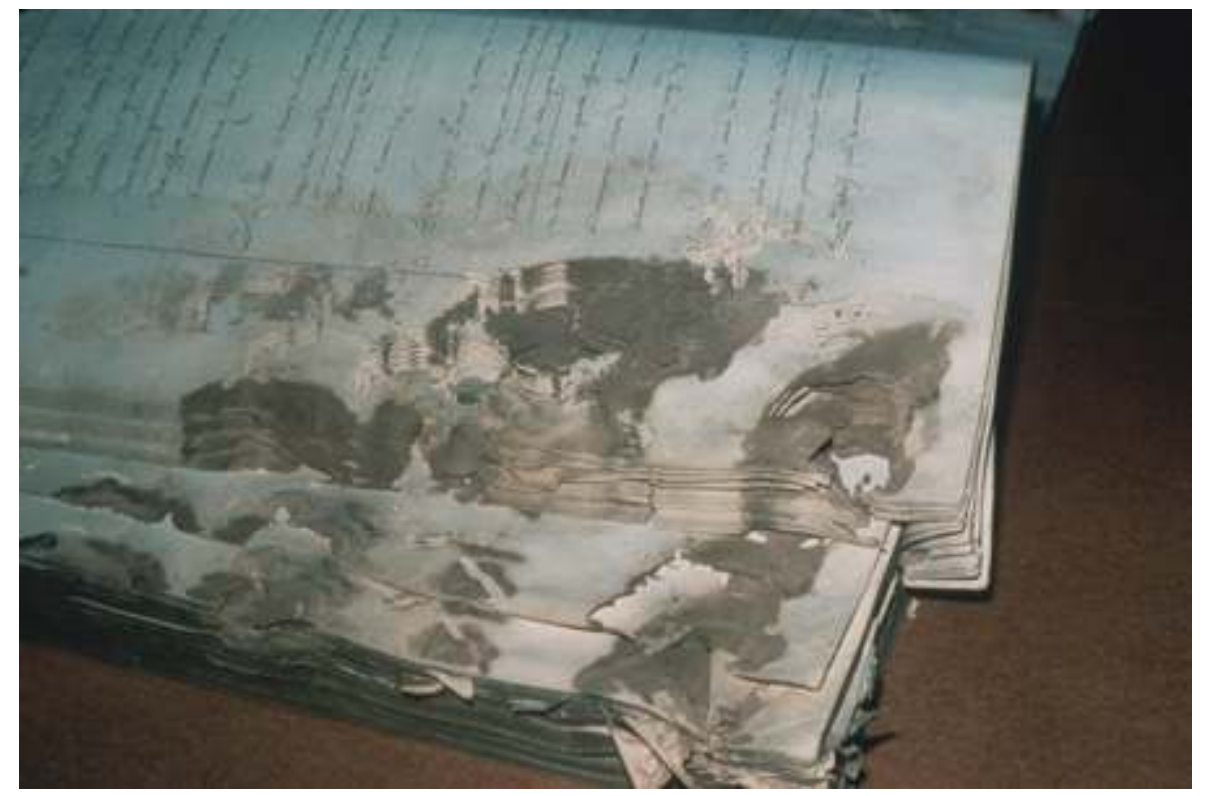

Papier altéré par l'acidité. @ CNRS Photothèque

Où écrire l'histoire de la biologie de la mémoire devient essentiel

Car tel devient alors le sens de toute l'histoire de la biologie de la mémoire : recherche de plus en plus fine des corrélats biologiques des différents types de mémoire révélés par la pathologie et les sciences cognitives, analyse de la possibilité de stockage dans le cerveau de différentes catégories d'information à l'échelle cellulaire et moléculaire, analyse des variations interindividuelles et interspécifiques des mécanismes cérébraux. À quoi s'ajoutent certains succès incontestables de l'imagerie cérébrale fonctionnelle à partir de la fin du $\mathrm{XX}^{\mathrm{e}}$ siècle. Les difficultés et les succès d'une telle entreprise, ne sauraient cependant faire oublier la profondeur historique de la notion de trace mémorielle, et masquer les tentatives d'élucidation, biologiquement périmées et néanmoins épistémologiquement instructives, de la mémoire avant les neurosciences. 


\section{BIBLIOGRAPHIE}

Dupont J.-C. Histoire de la neurotransmission, PUF, 1999.

Dupont J.-C., Schmitt S. Du feuillet au gène : une histoire de l'embryologie moderne, Éditions rue d'Ulm, 2004.

\section{NOTES}

1.Chapouthier G. Biologie de la mémoire, Odile Jacob, 2006. Kandel E. À la recherche de la mémoire, une nouvelle théorie de l'esprit, Odile Jacob, 2007. Schacter D. Science de la mémoire. Oublier et se souvenir, Odile Jacob, 2003.

2.Cf., par exemple, Foster J. K., Jelicic M. (eds). Memory: systems, process or function? Oxford University Press, 1999.

3.Colville-Stewart S. B., "Physicochemical models of the memory storage process: the historical role of arguments from analogy », PhD thesis, University of London, 1975 ; D. Draaisma, Metaphors of memory. A history of ideas about the mind, Cambridge University Press, 2000.

4.Krell D.F. Of memory, reminiscence, and writing, Indiana University Press, Bloomington and Indianapolis, 1990.

5.Sutton J. Philosophy and memory traces. Descartes to connectionism, Cambridge University Press, 1998.

6.Sur tout ce qui précède, cf. Dupont J.-C. (ed.) Histoires de la mémoire, Vuibert, 2005. 7.Chapouthier G. « Mémoires de mémoire », in La revue pour l'histoire du CNRS, n¹7, été 2007, pp. 32-35.

\section{RÉSUMÉS}

Sous sa forme individuelle chez l'homme, la mémoire signe des modifications biologiques au niveau cérébral susceptibles de diverses approches complémentaires. Jean-Claude Dupont nous livre des éléments d'épistémologie et d'histoire pour mieux comprendre cet objet de science.

Jean-Claude Dupont relates to the historical and epistemological relationship between biology and other disciplines (chemistry, physics, psychology and psychiatry), approached by the means of concepts located at their border, especially memory. 


\section{AUTEUR}

\section{JEAN-CLAUDE DUPONT}

Jean-Claude Dupont est maître de conférences en histoire et philosophie des sciences à l'université de Picardie et chercheur à l'Institut d'histoire et de philosophie des sciences (IHPST, ENS/Paris 1/CNRS UMR 8590). Ses recherches concernent l'histoire des neurosciences et de la biologie du développement. 